\title{
PREVALENCE OF CHRONIC ATRIAL FIBRILLATION IN DIALYSIS PATIENTS
}

By

James B. Wetmore

Submitted to the Graduate Degree Program in Preventive Medicine and Public Health and the Graduate Faculty of the University of Kansas in partial fulfillment of the requirements for the degree of Master of Science.

Chairperson: Theresa I. Shireman

Sally K. Rigler

Jonathan D. Mahnken

Date Defended: April 5, 2011 
The Thesis Committee for James B. Wetmore

certifies that this is the approved version for the thesis:

PREVALENCE OF CHRONIC ATRIAL FIBRILLATION IN DIALYSIS PATIENTS

Chairperson: Theresa I. Shireman

Date Approved: April 12, 2011 
Abstract

Background: Atrial fibrillation (AF) is reported to be common in patients on maintenance dialysis, but estimates of prevalence vary substantially. To date, no Medicare claims-based approach has been employed to rigorously assess prevalence of chronic AF.

Methods: A novel database was created to identify patients undergoing maintenance dialysis who were dually-eligible for Medicare and Medicaid for at least 3 months in 2004-05. A Medicare claims-based algorithm was used to generate a plausible range of chronic AF prevalences using four approaches. Poisson analysis was employed to determine the demographic, functional status, comorbidity, and other factors, as assessed by the Medical Evidence Form, associated with chronic AF.

Results: Of 102,748 dually-eligible individuals for whom data was complete, 21,540 (21.0\%) had at least one claim for non-perioperative AF. Raw percentages (irrespective of length of follow-up time) of individuals with chronic AF ranged from 9.8\% (using the most inclusive strategy) to $4.6 \%$ (the most exclusive); intermediate approaches led to closely-clustered estimates of $8.1 \%$ and $6.4 \%$. The intermediate approaches demonstrated chronic AF prevalence to range from 64.2 (95\% confidence intervals, 62.9 - 65.5) to $50.4(49.2-51.7)$ per 1000 patient-years. Age $>60$ years, male sex, Caucasian race, body mass index $>30$ $\mathrm{kg} / \mathrm{m}^{2}$, and inability to ambulate were associated with chronic AF; hypertension as a comorbidity was inversely associated with AF. Occurrence of AF was roughly 10 times greater in the youngest patients when compared to similar individuals not on dialysis. Conclusions: A linked Medicare-Medicaid database, together with a claims-based diagnostic algorithm, was used to generate prevalence estimates for chronic AF in dually-eligible dialysis patients. As expected, AF is far more common than in the non-dialysis population. 


\section{Acknowledgements:}

The author thanks Connie Wang, MD her for technical assistance with manuscript preparation, and the University of Kansas Clinical Research Curriculum Program grant K30 RR022275 (Edward Ellerbeck, PI).

\section{Sources of Funding:}

Funding for this study was provided by NIH grants R01 DK080111-02 (Theresa Shireman, PI) and K23 DK085378-01 (J.B.W.), by a National Kidney Foundation Young Investigator Award (J.B.W.), and by a Sandra A. Daugherty Foundation Grant (J.B.W.).

\section{Disclosures:}

The authors have no conflicts of interest to declare.

\section{Disclaimer:}

The data reported here have been supplied by the United States Renal Data System (DUA\#2007-10 \& 2009-19) and the Centers for Medicare \& Medicaid Services (DUA\#19707). The interpretation and reporting of these data are the responsibility of the author(s) and in no way should be seen as an official policy or interpretation of the U.S. government. 
Table of Contents

$\begin{array}{lc}\text { Abstract } & \text { iii } \\ \text { Acknowledgements } & \text { iv } \\ \text { Introduction } & 1 \\ \text { Methods } & 3 \\ \text { Results } & 9 \\ \text { Discussion } & 13 \\ \text { Figure } & 18 \\ \text { Tables } & 20 \\ \text { References } & 27\end{array}$




\section{Introduction}

Atrial fibrillation (AF) is common in end stage renal disease (ESRD) patients on maintenance dialysis, ${ }^{1-9}$ and is independently associated with mortality in the general ${ }^{10}$ and dialysis $^{8,11}$ populations. Recent studies examining the epidemiology of AF in cohorts drawn from a large dialysis-provider organization ${ }^{12}$ and from the Dialysis Outcomes and Practice Patterns Study (DOPPS) ${ }^{8}$ signal renewed interest in this area. Estimates of AF prevalence nevertheless remain quite variable, with rates in the literature having ranged from $6 \%{ }^{1}$ to nearly $27.0 \%{ }^{7,8}$ in certain circumstances. However, estimates provided by many studies are not directly comparable, and this relatively wide range is likely to reflect not only differences in individual study samples, but also in study design (e.g., prospective cohort vs. retrospective cohort vs. cross-sectional studies), classification of AF (e.g., truly chronic versus potentiallytransient), and ascertainment of the diagnosis (e.g., self-reports vs. targeted identification in longitudinal cohorts vs. retrospective records review). Additional work characterizing prevalence of chronic AF is therefore needed.

Development of a billing claims-based algorithm would provide additional opportunities for the study of AF. Such a validated algorithm has already proven extremely useful in non-dialysis patients with $\mathrm{AF}^{13,14}$, and use of a suitably-adapted algorithm in maintenance dialysis patients could allow a more far-reaching identification of dialysis patients with chronic AF. If combined with information on longitudinal medication exposures, studies of drug exposure-outcome relationships in dialysis patients with chronic AF could be undertaken in future. 
In this investigation, we constructed a novel linked Medicare-Medicaid database to determine prevalence of chronic AF in a large sample of maintenance dialysis patients. Using claims data provided by the United States Renal Data Service (USRDS), which incorporates Medicare data on dialysis patients, as well as 50-state Medicaid data, which permits ascertainment of filled prescription mediations, we identified dually-eligible (MedicareMedicaid) chronic dialysis patients in 2004-05. We then employed a rigorous claims-based algorithm, originally developed for identifying chronic AF in the general population ${ }^{13,14}$, to ascertain a range of plausible chronic AF prevalences in dialysis patients under various assumptions. Our aims were to provide a comprehensive examination of the prevalence of chronic AF in dually-eligible chronic U.S. dialysis patients, and to determine clinical and demographic factors associated with this disorder in a large cohort of dialysis patients. 


\section{Methods}

Study design

A retrospective cohort analysis of patients undergoing maintenance dialysis between January 1, 2004 and December 31, 2005 was utilized to identify persons with chronic AF; details are described below. The prevalence of risk factors for chronic AF were based on a cross-sectional analysis of those patients who were observable for at least 3 months during the 2-year window, after having initially survived their first 90 days on dialysis (thereby permitting inclusion in the USRDS).

\section{Data sources for analysis}

We utilized we data from three primary sources, the Centers for Medicare and Medicaid Services (CMS) Medicaid Analytic eXtract (MAX) files, Medicare Institutional and Physician/Supplier Claims files, and the USRDS. The USRDS tracks dialysis patients from initiation of dialysis through transplantation or death, and extracts Medicare data for end stage renal disease $(E S R D)$ patients enrolled in Medicare (comprising $\approx 98 \%$ of prevalent dialysis patients). USRDS data includes, from Medicare, the Institutional (“Part A”) and Physician/Supplier (“Part B”) Claims files. From CMS, the MAX Personal Summary (PS) files and final action prescription drug claim (Rx) files for calendar years 2004-05 were obtained. To link these sources, we created, from the MAX PS files, a record listing of unique Medicaid-eligible persons for all 50 states and D.C. based upon social security number (SSN), date of birth, gender, and state. For each unique record, we then created our own unique ID (“KUMC_ID”). This list was sent to the USRDS, which performed a series of 
deterministic matches against the USRDS core files. The USRDS removed SSNs and health insurance claim numbers (HICs), providing a crosswalk file that joined the USRDS_ID to the KUMC_ID. The USRDS_ID and KUMC_ID were thereby linked, enabling unique individuals to be identified. The KUMC_ID and USRDS_IDs were then linked to the appropriate MSIS_IDs within the MAX PS files, enabling further linkage (via the MSIS_ID) between the MAX PS and Rx files. We then replaced the MSIS_IDs with the USRDS_IDs, so that complete linkage was possible across Medicare Parts A \& B claims (previously provided by the USRDS) and the Medicaid PS \& Rx files.

To acquire requisite information on individual persons in the USRDS, the USRDS Core compact disk (CD) was used. Data contained in the Core CD is generated upon initiation of dialysis, when providers are required to submit to CMS the Medical Evidence Form (CMS 2728) documenting patient demographic characteristics, comorbid conditions, laboratory values prior to the first dialysis treatment, date of dialysis initiation, and dialysis modality and setting. Over time, changes in patient residence, payer status, and treatment history, as well as information on transplantation and death, are submitted to CMS and subsequently incorporated into the Core CD.

Study cohort and rationale for analytic approach

We identified unique individuals over the age of 20 years who survived $>90$ initial days on dialysis, who were Medicare-eligible for at least 90 days, and who were simultaneously enrolled in Medicaid and Medicare programs for at least 90 days during period the 2-year observation window. Medicare enrollment was verified using the USRDS PAYHIST files, while Medicaid eligibility was determined from the MAX PS files. We 
excluded persons who initiated HD prior to 1/1/1980, who were enrolled in the Veterans Administration healthcare system or in Medicaid managed care, and those who received a transplant prior to 1/1/2004. (Of note, persons with ESRD were not eligible for Medicare managed care plans prior to 2006.) We considered only the first period of dual eligibility (i.e., we censored at the first loss of dual eligibility).

\section{Determination of chronic atrial fibrillation}

We employed several rigorous algorithms adapted from one used by other investigators in order to determine the presence of chronic AF. The International Classification of Diseases (ICD-9) code 427.31 was used to identify $\mathrm{AF}^{13}$. To decrease the potential sources for misclassification attributable to transient, postoperative, valvular, or secondary sources of AF, exclusions were made using a method adapted from Go et $\mathrm{al}^{13,14}$. Specifically, we eliminated patients with evidence of valvular heart disease (from ICD-9 codes) and hyperthyroidism or thyrotoxicosis; exclusions for thyroid disorders were made based on the presence of relevant ICD-9 and/or CPT (Common Procedural Technology) and/or HCPCS (Healthcare Common Procedure Coding System) codes as well as prescriptions for propothiouracil or methimazole (identified from the MAX Rx files). In the case of potential perioperative sources of AF (e.g., coronary artery bypass surgery), claims (rather than individuals) were eliminated unless there was a preexisting ( $>30 \mathrm{~d}$ ) AF claim, and AF status determined as per the algorithm used for other individuals (described below). A full accounting of this exclusionary strategy listed in Supplementary Table 1.

To classify individuals as having chronic AF, we used a total of 4 algorithms, representing a spectrum of "liberal” to “conservative” approaches, in order to generate a range 
of plausible prevalence estimates. One cardinal approach utilized a total of 2 (or more) AF claims, at least 1 of which was an outpatient claim, and which had to be separated by 30 or more days. A second approach utilized a total of 3 (or more) AF claims (at least 2 of which were outpatient), in which each was separated by at least 30 days. To each of these two basic approaches, we added an additional element by establishing criteria for an "episode of care” window. Since outpatient AF claims related to a (subsequent) inpatient admission for AF could, conceivably, occur in the few days immediately prior to the hospital admission, we expunged all outpatient AF claims within 7 days of an AF claim-containing admission. Similarly, since outpatient followup care for AF could be closely tied to a recent hospital admission for AF, we expunged all outpatient AF claims within 30 days after an AF claimcontaining admission. By establishing the 7-day pre- and 30-day post-admission windows, AF claims likely to be associated with a single episode of care were eliminated. Our 4 approaches therefore consisted of a $2 \times 2$ matrix of claim number ( 2 claims versus 3 ) by episode-of-care window imposition (no window versus imposition of the 7 day-pre / 30 daypost window). Finally, to qualitatively compare or results to the larger dialysis population, we performed one additional analysis in which the full Medicare population (irrespective of Medicaid eligibility) was investigated, using the 2-claim approach with the episode-of-care window invoked.

Exposure to drugs used in the treatment of hyperthyroidism

Individuals who ever had a prescription for methimazole or propothiouracil were eliminated. MAX prescription drug claims included the national drug code (NDC) for these 
drugs $^{13,14}$. We matched drug name and therapeutic class information in the drug claims at the level of the NDC code using Multum Lexicon (Cerner Corporation, Kansas City, KS).

\section{Variables for analysis}

A variety of covariates, as recorded on the CMS 2728 form at time of dialysis initiation, were considered for analysis. Demographic variables were age, sex, and race by ethnicity (four mutually-exclusive groups consisting of non-Hispanic Caucasian, nonHispanic African-American, Hispanic, and Other). Body mass index (BMI) was classified into 4 categories; < $20 \mathrm{~kg} / \mathrm{m}^{2}, 20-24.99 \mathrm{~kg} / \mathrm{m}^{2}, 25-29.99 \mathrm{~kg} / \mathrm{m}^{2}, \geq 30 \mathrm{~kg} / \mathrm{m}^{2}$. Risk behavior factors examined were smoking and substance abuse (alcohol or illicit drugs), and functional status markers were employment, inability to ambulate, and inability to transfer. Major comorbidities were considered to be diabetes (types I and II combined), hypertension, congestive heart failure, coronary artery disease, cerebrovascular disease, peripheral vascular disease, and cardiac dysrhythmia. Since the CMS 2728 form is structured such that diseases like diabetes or hypertension may be considered as both a cause of ESRD and/or a comorbidity, diabetes and hypertension were considered present in an individual if they were listed as either the cause of ESRD or as a comorbidity ${ }^{15}$. Modality upon initiation of dialysis was categorized as in-center hemodialysis (HD) or self-care dialysis (home HD or peritoneal dialysis (PD)) based upon the dialysis modality history contained in the USRDS database. The sole laboratory value analyzed was hemoglobin at baseline, with a dichotomized level at $11 \mathrm{~g} / \mathrm{dL}$. Serums albumin was not analyzed, since $~ 20 \%$ of individuals did not have this value recorded. 
Statistical analyses: overall and specific approaches

We generated descriptive statistics (means for continuous variables and percentages for categorical variables) for the patients with and without chronic AF. To explore the differences between two groups (that is, those with and without chronic AF), we performed unconditional logistic regression for the bivariate analyses. Raw percentages of individuals who ever satisfied the definition of chronic AF (irrespective of length of followup time) were generated for each of the approaches. We then performed Poisson analysis in which the number of individuals with AF constituted the numerator and person-time of followup constituted the denominator; performance of the model was assessed by examination of residual plots. Age was dichotomized at 60 years to improve fit of the model. Adjusted prevalence rate ratios (APRRs) for AF were generated for each covariate.

Due to the large size of the study population, we attempt to distinguish between findings that were only statistically significant from those that are more likely to be clinically meaningful. Thus a $P$-value of $<0.01$ was considered the threshold for statistical significance. All statistical analyses were done with SAS 9.1 (Cary, NC, USA).

\section{Compliance and protection of human research participants}

The research protocol was approved by the institutional review board at the University of Kansas Medical Center (KUMC), and the project was undertaken according to the principles of the Declaration of Helsinki. Data Use Agreements (DUA) between KUMC and the USRDS and CMS permitted the data linking across the USRDS, Medicare and Medicaid files. In accordance with our DUA, social security numbers and other identifying information were removed from the linked files provided by CMS and the USRDS. 


\section{Results}

A total of 430,227 individuals on chronic dialysis were identified in the Core CDs from 2004 and 2005. Of these, 125,668 were dually-eligible (without managed care) for at least 90 days. Figure 1 demonstrates construction of the cohort. A small number of persons $(n=462)$ were excluded due to hyperthyroidism, while a larger number $(n=8384)$ were eliminated with evidence of valvular heart disease, leaving 116,858 persons. We then eliminated individuals with incomplete data, which left 102,748; the vast majority of attrition occurred because information on BMI and/or hemoglobin level was missing. Individuals with any claims for AF then numbered 21,780, but after eliminating claims within 30 days after a cardiac operation, 240 individuals were removed, leaving 21,540. This constitutes the initial numerator. Thus, $21.0 \%$ of persons had at least one claim for AF.

Supplementary Table 2 shows the distribution of inpatient (IP) and outpatient (OP) claims. Among the $85.9 \%$ who had no IP claims, $79.0 \%$ had no OP claims, 3.7\% one OP, $1.1 \%$ two OP, and $2.0 \%$ three or more OP claims. Of the $14.1 \%$ who had at least one IP claim, $2.0 \%$ had no OP claims, $1.5 \%$ one OP claim, $1.3 \%$ two OP claims, and $9.3 \%$ three or more OP claims.

Raw percentage estimates (that is, irrespective of length of followup) of persons with chronic AF were generated, as shown in Table 1. The most liberal approach (total of 2 or more claims, at least 1 of which was OP) yielded 9.8\%, while the most conservative (3 or more claims, at least 2 of which were OP, with the episode-of-care window restriction) yielded $4.6 \%$, a difference of roughly 2-fold. However, the intermediate approaches led to more closely-clustered estimates of $8.1 \%$ and $6.4 \%$, difference of about 1.3 -fold. 
Table 2 illustrates the bivariate analysis of the difference between patients with and without chronic AF of the characteristics upon initiation of dialysis of the; the 2-claim + episode-of-care window was used. The total cohort consisted of 102,748 individuals. Mean age was $59.4 \pm 15.5$ years and $52.6 \%$ were female, while $33.3 \%$ were Caucasian and $44.5 \%$ African-American. Fully 58.4\% were diabetics.

Of this cohort, 8347, or 8.1\%, had chronic AF. Patients with AF were significantly (P $<0.01$ ) more likely to be older (69.6 versus 58.6 years), female, Caucasian, have a higher BMI, be non-smokers and non-substance abusers, be unemployed, be unable to ambulate and transfer, and have diabetes (DM), congestive heart failure (CHF), coronary artery disease (CAD), peripheral vascular disease (PVD), and a history of a cerebrovascular accident (CVA). Patients with AF were also more likely to be on in-center hemodialysis and have a hemoglobin $(\mathrm{Hb})$ level $<11.0 \mathrm{~g} / \mathrm{dL}$. Only in the case of hypertension was the difference between persons with and without chronic AF less striking $(P=0.006)$, albeit still significant by our definition.

An analysis using the next-most conservative approach, namely the requirement of 3 or more claims but without the imposition of the episode-of-care window, yielded virtually identical results (not shown). The sole difference in the bivariate analysis using this approach was that hypertension was clearly no different between individuals with and without AF $(P=$ 0.15); all other previous associations remained significant.

Table 3 illustrates the result of Poisson regression modeling of the 2-claim, episode-of care approach. Age $>60 \mathrm{yr}$, male sex, Caucasian race, BMI $>30 \mathrm{~kg} / \mathrm{m}^{2}$, lack of smoking, unemployed status, inability to ambulate, HTN as the cause of ESRD, and histories of CAD, and CHF were significantly $(P<0.01)$ associated with chronic AF; history of CVA was 
nearly significant $(P=0.011)$. Lack of hypertension, considered as a comorbidity, was also associated with chronic AF. Specifically, the APRR of 0.82 indicates that, among those individuals in whom HTN was not the primary cause of ESRD, there was a lesser prevalence of chronic AF relative to those who did not have HTN as a comorbidity or a cause of ESRD. Notably, DM, use of self-care dialysis, and $\mathrm{Hb}<11 \mathrm{~g} / \mathrm{dL}$ were not associated with AF. Nearly-identical results were obtained in an analysis using the 3-claim approach without the episode-of-care window invoked (not shown), with the only difference being that employment status was no longer as significantly associated with $\operatorname{AF}(P=0.013)$.

To determine whether our analysis in dually-eligible patients was extrapolatable to the larger body of chronic dialysis patients, we ran a comparable analysis, using the 2-claim + episode-of-care approach, in the entire group of Medicare dialysis patients (i.e., irrespective of Medicaid eligibility). After all exclusions, there were 254,230 persons with complete data to study, with 61,782 having at least one AF claim, representing $24.3 \%$ of all individuals. The distribution pattern of claims originally depicted in Supplementary Table 1 was very similar in the entire group, with the only striking difference being that, in patients with at least 1 IP claim, $11.7 \%$ (versus $9.3 \%$ previously) had 3 or more OP claims. The overall raw percentage of individuals was higher, at $11.2 \%$, compared to the $8.1 \%$ of the comparable approach in dually-eligible patients. However, mean age of the patients in this group was much higher than in the dually-eligible group (63.3 versus 59.4 years, respectively). Poisson modeling in this group yielded generally similar results; all previously-identified associations remained intact, but inability to transfer, history of CVA, and use of in-center hemodialysis now became significant. A lack of hypertension as a comorbidity was once again associated with chronic AF. 
Formal prevalence estimates were generated from the Poisson model. For the duallyeligible patients using the 2-claim + episode-of-care window approach, the rate was 64.2 (95\% confidence intervals, 62.9 - 65.6) per 1000 person-years; for the 3-claim approach without the episode-of-care window, the rate was 50.4 (49.2 - 51.7) per 1000 person-years. In the older Medicare population, the rate (again using the 2-claim + episode-of-care window approach), the rate was $82.2(81.3$ - 83.2) per 1000 person-years.

Finally, to facilitate comparison with data in the general population, we calculated raw percentages of chronic AF by age, stratified by decade and sex, using the 2-claim + episodeof-care window approach. Table 4 displays these percentages. As expected, our estimate is higher than that of the general population and was evident across age strata. In males, absolute rates were roughly 2-fold (in the oldest individuals) to 10 -fold (in the youngest) higher in dialysis patients than in non-dialysis patients ${ }^{14}$. In young individuals aged $30-<40$, rates were fully $2.0 \%$ in females and $2.9 \%$ in males; in individuals aged $80-<90$, rates were $17.6 \%$ in females and $18.3 \%$ in males. These findings demonstrate that chronic AF both occurs at a younger age in the dialysis patients and has sustained high prevalence rates in the elderly. 


\section{Discussion}

In this study, we adapted a well-established Medicare claims-based algorithm for the identification of patients with chronic AF to the dually-eligible dialysis population. Our study compliments and expands upon other reports by demonstrating an approach through which claims data can be utilized for AF identification in dialysis patients, and by establishing a range of plausible prevalence estimates for this disorder. Since we believe it likely that our most liberal and conservative approaches overestimate and underestimate, respectively, prevalence of chronic AF, we suggest that the true prevalence likely lies between 50.4 and 64.2 per 1000 patient-years (between 6.4 and $8.1 \%$ in raw percentages), at least in duallyeligible U.S. dialysis patients. Since the general Medicare dialysis population is older than that with Medicaid, prevalence in the former is probably about 1.4 times higher. An additional contribution of the present report is quantification of the strong associations between chronic AF prevalence and a variety of demographic, anthropometric, risk behavior, functional status, comorbidity, dialysis modality, and laboratory-value factors as recorded upon dialysis initiation.

Given recent renewed interest in AF in dialysis patients ${ }^{8,9,12,16}$, the ability to identify such individuals using claims data provides a more universal platform for further investigation. This approach does not require utilization of more access-limited databases, such as those of large dialysis-provider organizations or of longitudinal epidemiological studies (e.g., DOPPS). Fortunately, a template for a claims-based algorithm for identification of AF exists, having been validated and published in a variety of widely-disseminated reports $^{13,14}$. However, we thought that some modification of the original approach was 
warranted, principally because dialysis patients utilize vastly greater healthcare resources, and, as a result, come into contact with the healthcare system far more often than do ambulatory patients with less prodigious comorbidity burdens ${ }^{17}$. We thus reasoned that imposing a restriction requiring an additional (i.e., $3^{\text {rd }}$ ) AF claim might result in less misclassification and would prove informative for one approach. Additionally, our overall strategy was informed by manual inspection of the claim patterns in several hundred patient records, which made it clear from a clinical perspective that some “outpatient” AF claims were almost certainly immediately associated with a proximate AF-related hospital admission. Therefore, establishment of an episode-of-care window paradigm, to minimize misclassification of acute as chronic AF, also seemed like a useful complementary approach. Fortunately, our two “intermediate” approaches (i.e., neither the most liberal nor the most conservative) led to prevalence estimates that were relatively close.

Our raw prevalence estimates appear to be somewhat lower than some ${ }^{6-8}$, but not all ${ }^{12}$, previous reports, but true prevalence comparisons may not be possible because we calculated events in person-time units using a Poisson analysis; most reports do not formally report rates as such. In terms of raw percentages, the DOPPS investigators recently reported a prevalence rate of $11.3 \%$ to $24.7 \%$ in Western countries, with the rate in the U.S. precisely reflecting the overall rate of $12.5 \%{ }^{8}$. However, once we empirically inspected the individual patterns of AF claims in several hundred patients in a variety of scenarios, many of previously-reported estimates seemed implausibly high to us. Recent work from a small but in-depth study of 256 patients revealed a lower-than-traditional estimate of $7.4 \%$ in incident dialysis patients, as well as overall rates of AF about $60 \%$ higher $(12.1 \%)^{11}$, suggesting that a substantial proportion of individuals labeled as having AF are likely to have transient AF or AF due to 
secondary causes. Thus high AF rates reported in other studies may be because individuals with secondary causes of AF or transient AF are being captured, and perhaps misclassified, by their respective identification strategies. Additional evidence for lower AF rates comes from a recent report by Chan et al, who concluded that AF rates were “only” $4.5 \%$ in incident dialysis patients ${ }^{12}$. It seems likely that rates would become higher in dialysis populations consisting of more “prevalent” individuals, since increasing age, a concomitant growing comorbidity burden, and increased exposure to the hemodialysis procedure itself (a physiologic stress test, given the need for rapid ultrafiltration) might be expected to result in increased rates of AF.

Our findings contribute to the understanding of the epidemiology of AF in part because the present study is larger than other studies by roughly an order of magnitude. As such, previously-reported findings of associations between various patient factors, at least those identified upon dialysis initiation, and chronic AF can be robustly quantified. As expected, we echoed the finding of the association of age with AF reported by Wizemann et al in the DOPPS database ${ }^{8}$, Abbott et al (in a univariate analysis) in Dialysis Morbidity and Mortality (DMMS) Wave II study ${ }^{18}$, Vasquez et al in their small but well-studied incident dialysis cohort $^{11}$, and others ${ }^{5,7}$. Both Abbott et al and Wizemann et al also found Caucasian race to be associated with $\mathrm{AF}^{8,18}$. The DOPPS investigators also found BMI to be significantly correlated in direct fashion with preexisting $\mathrm{AF}^{8}$. Notably, the role of sex in dialysis patients with AF remains unclear; while some investigators report sex to be unassociated with either incident or prevalent $\mathrm{AF}^{5,7,8}$, and others that female sex is the risk factor $^{11}$, our finding of an association of male sex with chronic AF is reflective of findings in 
the general population ${ }^{14}$. As anticipated, CAD and CHF emerged as comorbidity factors associated with AF in our study.

One interesting finding was an inverse association, after adjustment for other factors, between hypertension, considered as a comorbidity, and chronic AF in our study. Among individuals without HTN as their primary cause of ESRD, AF was less common in individuals with HTN as a comorbidity than in those without. While HTN is a risk factor for AF in the general population ${ }^{19-24}$, in ESRD, studies of prevalent dialysis patients may be characterized by a distinct physiology, namely the controversially-termed phenomenon of “reverse epidemiology”25, 26 . Evidence from the DOPPS database appears to support our results: while a formal diagnosis of hypertension was not associated with AF, low pre-dialysis systolic blood pressure was $^{8}$, lending plausibility to the hypothesis that when dialysis patients manifest AF, many of them are experiencing decreased cardiac output and concomitant hypotension. Since AF may contribute to hypotension in dialysis patients, this might be an explanation for our findings.

Our study has several important limitations. First, we did not have access to medical charts. However, we adapted a well-established validated algorithm with the express goal of minimizing the possibility that secondary, transient, or postoperative causes of AF would be misclassified as truly chronic AF. We then performed a series of increasingly-conservative approaches to yield an overall range of plausible prevalence estimates. Second, we used the CMS 2728 form to identify comorbidities, as is widely done ${ }^{15,27-32}$. Although this form has several strengths, a more rigorous, but far more complex, approach would be to use a claimsbased approach to identify comorbidities. Such an approach exists ${ }^{33,34}$, and should be a subject of future investigations, but it does require a minimum survival of 9 months and is 
therefore could introduce an immortal time bias ${ }^{35}$; any conclusions drawn from such an approach would have to be considered accordingly. Finally, our primary analysis was confined to dually-eligible individuals. Since individuals with Medicaid are likely to be the most medically-needy and to have more comorbidities at a younger age than the non-duallyeligible population ${ }^{36}$, our results can only be generalized with caution. However, we performed an additional analysis in the general Medicare population, and found a consistent estimate once the increased age of non-Medicaid individuals is considered.

In conclusion, we used a Medicare claims-based approach to define the prevalence of chronic AF in a large number of dually-eligible maintenance dialysis patients. This approach is likely to prove suitable for future investigations using Medicare and Medicaid data. Our prevalence estimate is somewhat lower than that of many reports, which is probably due to the rigor of our strategy for identifying chronic AF. Even so, the prevalence rates we report are still far higher than in the non-dialysis population. The important factors and comorbidities associated with chronic AF generate hypotheses suitable for future study. Investigations of many aspects of chronic AF in dialysis patients, such as further examinations of the patterns of warfarin use and the risks and benefits of this therapy, should be undertaken. 
Figure 1. Exclusion flowchart demonstrating the creation of the cohort of individuals without claims for atrial fibrillation ("denominator") and with at least one non-perioperative claim for atrial fibrillation (“numerator”) for 2004-05. 
1.

Dually-eligible for Medicare and Medicaid (without managed care) for $\geq 90$ days

$$
N=125,668
$$

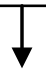

Excluded* due to hyperthyroidism (ICD-9, CPT, Medicaid Rx records): $\mathbf{N}=\mathbf{4 6 2}$

Excluded* due to valvular heart disease: $\mathbf{N}=\mathbf{8 3 8 4}$

$$
\mathbf{N}=\mathbf{1 1 6 , 8 5 8} \text { remaining }
$$

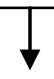

Excluded due to incomplete data: $\mathrm{N}=14,110$

$$
\mathbf{N}=102,748 \text { remaining }
$$

(“denominator”)

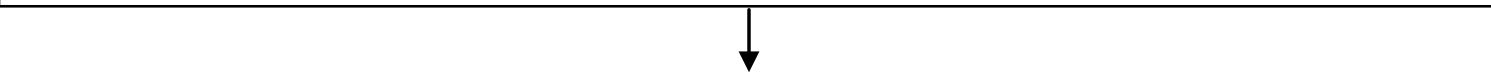

Individuals with at least one claim for AF

$$
\mathbf{N}=\mathbf{2 1 , 7 8 0} \text { remaining }
$$

$$
\begin{aligned}
& \text { Excluded due to claims being related to cardiac surgery: } \mathbf{N}=\mathbf{2 4 0} \\
& \qquad \mathbf{N}=\mathbf{2 1 , 5 4 0} \text { remaining } \\
& \text { ("numerator" of individuals with at least } 1 \text { claim for non-perioperative AF) }
\end{aligned}
$$

*Some individuals excluded due to both hyperthyroidism and valvular heart disease.

ICD-9, International Classification of Diseases-9; CPT, Common Procedural Terminology; HCPCS, Healthcare Common Procedural Coding System; Rx, prescription; AF, atrial fibrillation. 
Table 1. Sources of exclusion of individuals with potential secondary sources of atrial fibrillation.

\begin{tabular}{|c|c|c|c|}
\hline Variable & Source & Exclusion Level & Time Period \\
\hline Coronary bypass surgery & ICD-9 36.10-.19 & claim & within 30 days of $\mathrm{AF}$ \\
\hline $\begin{array}{l}\text { Cardiac surgery/ } \\
\text { procedure }\end{array}$ & $\begin{array}{l}\text { ICD-9 35.31-.39, .41- } \\
.42, .50-.54, .60-.63, .70- \\
.73,37.24-.25, .35\end{array}$ & claim & within $30 \mathrm{~d}$ \\
\hline Valvular repair & $\begin{array}{l}\text { ICD-9 35.01-.02, .11- } \\
.12, .21-.24\end{array}$ & claim & within $30 \mathrm{~d}$ \\
\hline Valvular heart disease & $\begin{array}{l}\text { ICD-9 394.0, .2, 396.0- } \\
.1, .8,746.5, \text { V42.2, } \\
\text { V43.3 }\end{array}$ & person & ever \\
\hline Pericardial surgery & $\begin{array}{l}\text { ICD-9 37.10-.12, .31- } \\
.33, .40\end{array}$ & claim & within $30 \mathrm{~d}$ \\
\hline $\begin{array}{l}\text { Hyperthyroidism/ } \\
\text { thyrotoxicosis }\end{array}$ & $\begin{array}{l}\text { ICD-9 242.0-.9; CPT } \\
\text { 2420-24, 2428-29; } \\
\text { HCPCS 79000-1, 79005; } \\
\text { propylthiouracil or } \\
\text { methimazole }\end{array}$ & person & ever \\
\hline
\end{tabular}

ICD-9, International Classification of Diseases-9; AF, atrial fibrillation; CPT, Common Procedural Technology; HCPCS, Healthcare Common Procedure Coding System 
Table 2. Percentage distribution of outpatient and inpatient claims for atrial fibrillation in dually-eligible chronic dialysis patients, 2004-05.

\begin{tabular}{lccccc}
\hline & 0 Outpatient & 1 Outpatient & 2 Outpatient & 3+ Outpatient & Total \\
\hline 0 Inpatient,\% & 79.0 & 3.7 & 1.1 & 2.0 & 85.9 \\
1+ Inpatient, \% & 2.0 & 1.5 & 1.3 & 9.3 & 14.1 \\
\hline
\end{tabular}


Table 3. Percentage of individuals with chronic atrial fibrillation, according to the four definitions.

\begin{tabular}{lc}
\hline Identification strategy & \% with AF \\
\hline 2+ claims & 9.8 \\
2+ claims with episode-of-care window & 8.1 \\
3+ claims & 6.4 \\
3+ claims with episode-of-care window & 4.6 \\
\hline In the case of individuals with 2 claims, no more than 1 \\
could be an inpatient claim. In the case of individuals \\
with 3 claims, no more than 1 could be an inpatient claim. \\
"Episode of care window" defined in text. AF, chronic \\
atrial fibrillation
\end{tabular}


Table 4. Characteristics of dialysis patients with and without chronic atrial fibrillation.

\begin{tabular}{|c|c|c|c|c|}
\hline Characteristic & $\begin{array}{c}\text { Total } \\
(n=102748)\end{array}$ & $\begin{array}{c}\mathrm{AF} \\
(n=8347)\end{array}$ & $\begin{array}{c}\text { non-AF } \\
(n=94401)\end{array}$ & $P$-value \\
\hline Age, years & $59.4 \pm 15.5$ & $69.6 \pm 11.5$ & $58.6 \pm 15.4$ & $<0.0001$ \\
\hline Female sex, $n(\%)$ & $54016(52.6)$ & 4717 (56.5) & 49299 (52.2) & $<0.0001$ \\
\hline Race/Ethnicity, $n$ (\%) & & & & $<0.0001$ \\
\hline African-American & $45751(44.5)$ & $2646(31.7)$ & 43105 (45.7) & \\
\hline Caucasian & 34258 (33.3) & $4209(50.4)$ & 30049 (31.8) & \\
\hline Hispanic & $16443(16.0)$ & 967 (11.6) & $15476(16.4)$ & \\
\hline Other & $6296(6.1)$ & $525(6.3)$ & $5771(6.1)$ & \\
\hline BMI category, $n(\%)$ & & & & $<0.0001$ \\
\hline$<20 \mathrm{~kg} / \mathrm{m}^{2}$ & $9819(9.6)$ & $699(8.4)$ & $9120(9.7)$ & \\
\hline $20-24.9 \mathrm{~kg} / \mathrm{m}^{2}$ & $29805(29.0)$ & $2223(26.6)$ & $27582(29.2)$ & \\
\hline $25-29.9 \mathrm{~kg} / \mathrm{m}^{2}$ & $27952(27.2)$ & $2290(27.4)$ & $25662(27.2)$ & \\
\hline $30+\mathrm{kg} / \mathrm{m}^{2}$ & $35172(34.2)$ & 3135 (37.6) & 32037 (33.9) & \\
\hline Smoker, $n(\%)$ & $6819(6.6)$ & $382(4.6)$ & $6437(6.8)$ & $<0.0001$ \\
\hline $\begin{array}{l}\text { Substance abuser, } n \\
(\%)\end{array}$ & 3345 (3.3) & $140(1.7)$ & 3205 (3.4) & $<0.0001$ \\
\hline Unemployed, $n(\%)$ & $96290(93.7)$ & $8106(97.1)$ & $88184(93.4)$ & $<0.0001$ \\
\hline $\begin{array}{l}\text { Inability to ambulate, } \\
n(\%)\end{array}$ & $4226(4.1)$ & $563(6.7)$ & 3663 (3.9) & $<0.0001$ \\
\hline Inability to transfer, $n$ & $1487(1.4)$ & $227(2.7)$ & $1260(1.3)$ & $<0.0001$ \\
\hline $\begin{array}{l}\text { Primary cause of } \\
\text { ESRD, } n(\%)\end{array}$ & & & & $<0.0001$ \\
\hline Diabetes & $50670(49.3)$ & 4199 (50.3) & $46471(49.2)$ & \\
\hline Hypertension & $27805(27.1)$ & $2381(28.5)$ & 25424 (26.9) & \\
\hline Glomerulonephritis & $9892(9.6)$ & $539(6.5)$ & 9353 (9.9) & \\
\hline Other & $14381(14.0)$ & $1228(14.7)$ & 13153 (13.9) & \\
\hline \multicolumn{5}{|l|}{ Comorbidities, $n$ (\%) } \\
\hline Hypertension & 87705 (85.4) & 7039 (84.3) & 80666 (85.5) & 0.006 \\
\hline Diabetes & $59973(58.4)$ & $5138(61.6)$ & $54835(58.1)$ & $<0.0001$ \\
\hline $\mathrm{CHF}$ & 29718 (28.9) & 3715 (44.5) & $26003(27.5)$ & $<0.0001$ \\
\hline CAD & $20630(20.1)$ & 2819 (33.8) & $17811(18.9)$ & $<0.0001$ \\
\hline PVD & $12139(11.8)$ & $1419(17.0)$ & $10720(11.4)$ & $<0.0001$ \\
\hline CVA & $9100(8.9)$ & $1023(12.3)$ & $8077(8.6)$ & $<0.0001$ \\
\hline $\begin{array}{l}\text { Self-care dialysis, } n \\
(\%)\end{array}$ & $5742(5.6)$ & $364(4.4)$ & $5378(5.7)$ & $<0.0001$ \\
\hline $\begin{array}{l}\mathrm{Hb}<11.0 \mathrm{~g} / \mathrm{dL}, n \\
(\%)\end{array}$ & 79061 (76.9) & $6271(75.1)$ & $72790(77.1)$ & $<0.0001$ \\
\hline
\end{tabular}


AF, chronic atrial fibrillation; BMI, body mass index; ESRD, end stage renal disease; $\mathrm{CHF}$, congestive heart failure; CAD, coronary artery disease; PVD, peripheral arterial disease; CVA, cerebrovascular accident; HD, hemodialysis; Hb, hemoglobin 
Table 5. Adjusted prevalence rate ratio estimates for dialysis patients with chronic atrial fibrillation.

\begin{tabular}{|c|c|c|c|}
\hline Characteristic & APRR & 95\% CIs & $P$-value \\
\hline Age $>60 y$ & 3.37 & $3.18-3.57$ & $<0.0001$ \\
\hline Male Sex & 1.12 & $1.08-1.18$ & $<0.0001$ \\
\hline \multicolumn{4}{|l|}{ Race/Ethnicity } \\
\hline Caucasian & - & - & - \\
\hline African-American & 0.50 & $0.48-0.53$ & $<0.0001$ \\
\hline Hispanic & 0.45 & $0.42-0.49$ & $<0.0001$ \\
\hline Other & 0.64 & $0.59-0.71$ & $<0.0001$ \\
\hline \multicolumn{4}{|l|}{ BMI category } \\
\hline $20-24.9 \mathrm{~kg} / \mathrm{m}^{2}$ & - & - & - \\
\hline$<20 \mathrm{~kg} / \mathrm{m}^{2}$ & 0.98 & $0.90-1.07$ & 0.69 \\
\hline $25-29.9 \mathrm{~kg} / \mathrm{m}^{2}$ & 1.06 & $1.01-1.13$ & 0.043 \\
\hline $30+\mathrm{kg} / \mathrm{m}^{2}$ & 1.22 & $1.16-1.29$ & $<0.0001$ \\
\hline Smoker, $n$ (\%) & 0.80 & $0.72-0.89$ & $<0.0001$ \\
\hline Substance abuser, $n(\%)$ & 0.88 & $0.74-1.05$ & 0.15 \\
\hline Employed & 0.80 & $0.70-0.91$ & 0.0009 \\
\hline Inability to ambulate & 1.19 & $1.07-1.32$ & 0.0015 \\
\hline Inability to transfer & 1.21 & $1.03-1.43$ & 0.020 \\
\hline \multicolumn{4}{|l|}{ Comorbidities } \\
\hline Hypertension & 0.82 & $0.77-0.87$ & $<0.0001$ \\
\hline Diabetes & 1.01 & $0.94-1.09$ & 0.82 \\
\hline CAD & 1.29 & $1.22-1.35$ & $<0.0001$ \\
\hline $\mathrm{CHF}$ & 1.53 & $1.46-1.60$ & $<0.0001$ \\
\hline CVA & 1.09 & $1.02-1.17$ & 0.011 \\
\hline PVD & 1.02 & $0.96-1.08$ & 0.54 \\
\hline \multicolumn{4}{|l|}{ Cause of ESRD } \\
\hline Diabetes & - & - & - \\
\hline Hypertension & 1.30 & $1.21-1.41$ & $<0.0001$ \\
\hline Glomerulonephritis & 1.08 & $0.97-1.21$ & 0.17 \\
\hline Other & 1.14 & $1.04-1.24$ & 0.0039 \\
\hline Self-care dialysis & 0.94 & $0.85-1.05$ & 0.29 \\
\hline $\mathrm{Hb}<11.0 \mathrm{~g} / \mathrm{dL}$ & 0.98 & $0.94-1.03$ & 0.53 \\
\hline
\end{tabular}

APRR, adjusted prevalence ratio ratio; CI, confidence interval; BMI, body mass index; ESRD, end stage renal disease; CHF, congestive heart failure; CAD, coronary artery disease; PVD, peripheral arterial disease; CVA, cerebrovascular accident; HD, hemodialysis; Hb, hemoglobin 
Table 6. Percentage of individuals identified as having chronic atrial fibrillation, by age decade and sex.

\begin{tabular}{ccc}
\hline Age decade & F with AF,\% & M with AF,\% \\
\hline $20-29.9$ & 0.6 & 0.8 \\
$30-39.9$ & 1.2 & 1.4 \\
$40-49.9$ & 2.0 & 2.9 \\
$50-59.9$ & 5.0 & 5.3 \\
$60-69.9$ & 8.7 & 9.7 \\
$70-79.9$ & 14.2 & 15.5 \\
$80-89.9$ & 17.6 & 18.3 \\
$90+$ & 18.3 & 19.7 \\
\hline
\end{tabular}

$\mathrm{AF}$, chronic atrial fibrillation 


\section{References}

1. Foley RN, Parfrey PS, Harnett JD, et al. Clinical and echocardiographic disease in patients starting end-stage renal disease therapy. Kidney Int. Jan 1995;47(1):186-192.

2. United States Renal Data System. USRDS 2005 Annual Data Report: Atlas of EndStage Renal Disease in the United States. In: National Institutes of Health, National Institute of Diabetes and Digestive and Kidney Diseases. 2005.

3. Abe S, Yoshizawa M, Nakanishi N, et al. Electrocardiographic abnormalities in patients receiving hemodialysis. Am Heart J. Jun 1996;131(6):1137-1144.

4. Vazquez E, Sanchez-Perales C, Borrego F, et al. Influence of atrial fibrillation on the morbido-mortality of patients on hemodialysis. Am Heart J. Dec 2000;140(6):886890.

5. Atar I, Konas D, Acikel S, et al. Frequency of atrial fibrillation and factors related to its development in dialysis patients. Int J Cardiol. Jan 4 2006;106(1):47-51.

6. Fabbian F, Catalano C, Lambertini D, et al. Clinical characteristics associated to atrial fibrillation in chronic hemodialysis patients. Clin Nephrol. Sep 2000;54(3):234-239.

7. Genovesi S, Pogliani D, Faini A, et al. Prevalence of atrial fibrillation and associated factors in a population of long-term hemodialysis patients. Am J Kidney Dis. Nov 2005;46(5):897-902.

8. Wizemann V, Tong L, Satayathum S, et al. Atrial fibrillation in hemodialysis patients: clinical features and associations with anticoagulant therapy. Kidney Int. Jun;77(12):1098-1106.

9. Korantzopoulos P, Kokkoris S, Liu T, Protopsaltis I, Li G, Goudevenos JA. Atrial fibrillation in end-stage renal disease. Pacing Clin Electrophysiol. Nov 2007;30(11):1391-1397.

10. Benjamin EJ, Wolf PA, D'Agostino RB, Silbershatz H, Kannel WB, Levy D. Impact of atrial fibrillation on the risk of death: the Framingham Heart Study. Circulation. Sep 8 1998;98(10):946-952.

11. Vazquez E, Sanchez-Perales C, Garcia-Garcia F, et al. Atrial fibrillation in incident dialysis patients. Kidney Int. Aug 2009;76(3):324-330.

12. Chan KE, Lazarus JM, Thadhani R, Hakim RM. Warfarin use associates with increased risk for stroke in hemodialysis patients with atrial fibrillation. J Am Soc Nephrol. Oct 2009;20(10):2223-2233.

13. Go AS, Hylek EM, Borowsky LH, Phillips KA, Selby JV, Singer DE. Warfarin use among ambulatory patients with nonvalvular atrial fibrillation: the anticoagulation and risk factors in atrial fibrillation (ATRIA) study. Ann Intern Med. Dec 21 1999;131(12):927-934.

14. Go AS, Hylek EM, Phillips KA, et al. Prevalence of diagnosed atrial fibrillation in adults: national implications for rhythm management and stroke prevention: the AnTicoagulation and Risk Factors in Atrial Fibrillation (ATRIA) Study. Jama. May 9 2001;285(18):2370-2375.

15. Volkova N, McClellan W, Soucie JM, Schoolwerth A. Racial disparities in the prevalence of cardiovascular disease among incident end-stage renal disease patients. Nephrol Dial Transplant. Aug 2006;21(8):2202-2209. 
16. Korantzopoulos PG, Goudevenos JA. Atrial fibrillation in end-stage renal disease: an emerging problem. Kidney Int. Aug 2009;76(3):247-249.

17. Foley RN, Collins AJ. End-stage renal disease in the United States: an update from the United States Renal Data System. J Am Soc Nephrol. Oct 2007;18(10):2644-2648.

18. Abbott KC, Trespalacios FC, Taylor AJ, Agodoa LY. Atrial fibrillation in chronic dialysis patients in the United States: risk factors for hospitalization and mortality. BMC Nephrol. Jan 24 2003;4:1.

19. Wilson PW, D'Agostino RB, Levy D, Belanger AM, Silbershatz H, Kannel WB. Prediction of coronary heart disease using risk factor categories. Circulation. May 12 1998;97(18):1837-1847.

20. Executive Summary of The Third Report of The National Cholesterol Education Program (NCEP) Expert Panel on Detection, Evaluation, And Treatment of High Blood Cholesterol In Adults (Adult Treatment Panel III). Jama. May 16 2001;285(19):2486-2497.

21. Chambless LE, Folsom AR, Sharrett AR, et al. Coronary heart disease risk prediction in the Atherosclerosis Risk in Communities (ARIC) study. J Clin Epidemiol. Sep 2003;56(9):880-890.

22. Folsom AR, Chambless LE, Duncan BB, Gilbert AC, Pankow JS. Prediction of coronary heart disease in middle-aged adults with diabetes. Diabetes Care. Oct 2003;26(10):2777-2784.

23. Schnabel RB, Sullivan LM, Levy D, et al. Development of a risk score for atrial fibrillation (Framingham Heart Study): a community-based cohort study. Lancet. Feb 28 2009;373(9665):739-745.

24. Chamberlain AM, Agarwal SK, Folsom AR, et al. A clinical risk score for atrial fibrillation in a biracial prospective cohort (from the Atherosclerosis Risk in Communities [ARIC] study). Am J Cardiol. Jan;107(1):85-91.

25. Kovesdy CP, Kalantar-Zadeh K. Introduction: the reverse epidemiology controversy. Semin Dial. Nov-Dec 2007;20(6):485.

26. Kalantar-Zadeh K. What is so bad about reverse epidemiology anyway? Semin Dial. Nov-Dec 2007;20(6):593-601.

27. Kramer HJ, Saranathan A, Luke A, et al. Increasing body mass index and obesity in the incident ESRD population. J Am Soc Nephrol. May 2006;17(5):1453-1459.

28. Trivedi H, Xiang Q, Klein JP. Risk factors for non-fatal myocardial infarction and cardiac death in incident dialysis patients. Nephrol Dial Transplant. Jan 2009;24(1):258-266.

29. Hirth RA, Turenne MN, Wheeler JR, Pan Q, Ma Y, Messana JM. Provider monitoring and pay-for-performance when multiple providers affect outcomes: An application to renal dialysis. Health Serv Res. Oct 2009;44(5 Pt 1):1585-1602.

30. Foley RN, Chen SC, Collins AJ. Hemodialysis access at initiation in the United States, 2005 to 2007: still "catheter first". Hemodial Int. Oct 2009;13(4):533-542.

31. Foley RN, Roberts TL, Liu J, et al. Mortality from cancer among US hemodialysis patients, 1995-2005. Am J Nephrol.31(6):518-526.

32. Dalrymple LS, Johansen KL, Chertow GM, et al. Infection-related hospitalizations in older patients with ESRD. Am J Kidney Dis. Sep;56(3):522-530. 
33. Liu J, Huang Z, Gilbertson DT, Foley RN, Collins AJ. An improved comorbidity index for outcome analyses among dialysis patients. Kidney Int. Jan;77(2):141-151.

34. Seliger SL. Comorbidity and confounding in end-stage renal disease. Kidney Int. Jan;77(2):83-85.

35. Suissa S. Effectiveness of inhaled corticosteroids in chronic obstructive pulmonary disease: immortal time bias in observational studies. Am J Respir Crit Care Med. Jul 1 2003;168(1):49-53.

36. Wetmore JB, Rigler SK, Mahnken JD, Mukhopadhyay P, Shireman TI. Considering health insurance: how do dialysis initiates with Medicaid coverage differ from persons without Medicaid coverage? Nephrol Dial Transplant. Jan;25(1):198-205. 